\title{
Facial Expression Recognition Based on Basic Expressions and Intensities Using K-Means Clustering
}

\author{
Rohit Pal ${ }^{1}$, Dr. C. S. Satsangi ${ }^{2}$ \\ ${ }^{1}$ ME Scholar, IT Department, Medicaps Institute of Technology and Management, Indore (M.P), India \\ ${ }^{2}$ Professor, IT Department, Medicaps Institute of Technology and Management, Indore (M.P), India
}

\begin{abstract}
Facial expression recognition provides rich information for human-robot interaction with respect to emotions. This paper presents a facial expression recognition method that recognizes facial expressions as well as intensity and mixture ratio of basic facial expressions. In this system, K-means clustering method is applied on Cohn-Kanade image database. Algorithms are adopted to align the input facial images to get texture features. A novel method is proposed to recognize mixture ratio of very basic facial expressions which comprises of 6 facial expressions viz. happy, angry, fear, neutral, sad and surprise and also the intensity of the expression. Back propagation algorithm is used to obtain the recognition scores, which are then used again to classify the facial expression results. Experimental results verified that the proposed method can effectively recognize the image and expression intensity and provides an accuracy of $98 \%$.
\end{abstract}

Keywords: K mean Clustering, Back Propagation, Mixture Ratio, Facial Expression Coding System (FACS), Facial Expression Intensity, Confusion Matrix

\section{Introduction}

Image processing is a technique to translate an image into digital form and execute some operations on it, in order to get an improved/enhanced image or to extract some useful information from it. It is a type of signal indulgence in which input is image, like photograph or video frame and output may be an image or characteristics associated with that image. Basically Image Processing scheme includes treating images as two dimensional signals whilst applying already set signal processing methods to them. As a computer-based technology, digital image processing carries out automatic processing, manipulation and interpretation of such visual information, and it plays a very important role in various aspects of our daily life, as well as in a wide diversity of disciplines and fields in science and technology, with applications such as television, robotics, remote sensing, medical diagnosis, remote sensing and industrial inspection. Some of the areas in which it is useful is Space image processing (interplanetary probe images), Medical/Biological image processing, Automatic character recognition etc. There are different types of tasks performed in digital image processing which includes Image Acquisition, storage, transmission, Image Enhancement and restoration, Image understanding and recognition.

\section{Literature Review}

Kai Tai Song and Shuo-Cheng Chien et al. proposed a facial recognition technique which is based on mixture of basic facial expressions and intensities. In this technique, Active Appearance Model (AAM) and Lucas-Kanade image alignment algorithms are adopted to line up the key facial images to attain texture features. Three kinds of texture features are used in this method which comprises of texture features of the whole face, which are used as inputs of facial expression intensity recognition; secondly the texture features of the upside face, which are used as inputs of upper face action units recognition; and lastly texture features of the downside face, which are used as the inputs of lower face action units recognition. Back propagation neural networks are used to attain the recognition scores, which are then oppressed to organize the facial expression results. The major limitation of this technique is that a lot of complex methodologies are involved thereby making it very lengthy method to reach at the result. Also the accuracy achieved which is $93.3 \%$ for basic expression recognition and $95.6 \%$ for expression intensity experiment is quite low in terms of the efforts.

Mingwei Huang, Zhen Wang and Zilu Ying introduced a method for facial recognition which is based on stochastic neighbor embedding and support vector machines. Now as the method uses the above two mentioned techniques, therefore it would not be wrong to say that it is relying on the predictive techniques to recognize an expression which could minimize the efficiency of the method to some extent. The major problem of stochastic based method is that it uses probabilistic approach which increases the risk of not recognizing a facial expression and thereby increasing the further risk of not recognizing an image. The second shortcoming of this method is that the database used here is Japanese Female Facial Expression (JAFFE) database which is again indicating that it is confined in this particular area that is no generalized database is used.

Kai-Tai Song and Yi-Wen Chen proposed a novel integrated technique for facial recognition. In this technique facial images from a web camera are first taken for facial shape and texture model production using active appearance model. After then Modified Lucas-Kanade image alignment algorithm is taken into consideration to find facial features as well as the texture model of AAM to build facial texture parameters. A novel design is projected for an integrated 


\section{International Journal of Science and Research (IJSR) \\ ISSN (Online): 2319-7064}

Index Copernicus Value (2013): 6.14 | Impact Factor (2014): 5.611

facial expression detection system. In the very first stage, face detection is carried out to find user's identity; then the facial-expression database of the recognized user is engaged to recognize his/her facial expressions. These parameters are utilized to coach the back propagation neural networks (BPNN) for face and facial expression recognition. One major limitation which has come out is that it uses 30 images per person in order to train the system which is quite large its own also the average face recognition percentage is $96.7 \%$ which is low as far as number of images are concerned.

Huiquan Zhang, Sha Luo and Osamu Yoshie proposed an approach for facial expression by finding associations amid visual feature and Local Binary Pattern(LBP). Unlike many prior studies, the proposed approach by design tracks the facial area and segments face into important areas based on description of Local Binary Pattern and then it accumulates the probabilities throughout the frames from video data to confine the temporal characteristics of facial expressions by analyzing various expressions. Through this approach, the chronological variation of facial expression can be quantified in personage areas. Therefore, the process of recognizing facial expression tends to be more logical without sacrificing results of recognition. Though there are many advantages in the proposed algorithm but the probabilistic approach equalizes them all. As mentioned earlier it is quite riskier to rely on this approach also it relies on unsupervised learning technique.

Nazil Perveen, Shubhrata Gupta, and Kesari Verma introduced a new technique for facial expression recognition using facial characteristics points and gini index. This paper focuses on the fact that facial expression information is mainly concentrated on facial expression information regions, so the, eyes, eyebrows, and mouth are extracted from the input image. When a face image is entered as input, the feature extraction is performed which helps in detecting facial characteristic points. The proposed technique is applied to JAFFE database consisting 30 images, each having 6 very basic facial expression images (neutral, surprise, happy, fear, angry and sad). The input image template is $256 * 256$ pixels, The eye template is $15 * 30$ pixels, the eyebrow template is $15 * 40$ and the mouth template is $25 * 40$ pixels. In the first step the input is given in the form of target image and template. These are then converted into gray scale images after which a 2-D convolution of the target image and template image are found. The mean and variance of the template image is then calculated and then the correlation score is calculated which is matched in order to complete the search. The major drawback of this technique is the accuracy and the database used which is JAFFE thereby confining it to a small domain.

\section{Proposed Scheme}

The proposed algorithm is shown in the form of flow chart and is explained below:

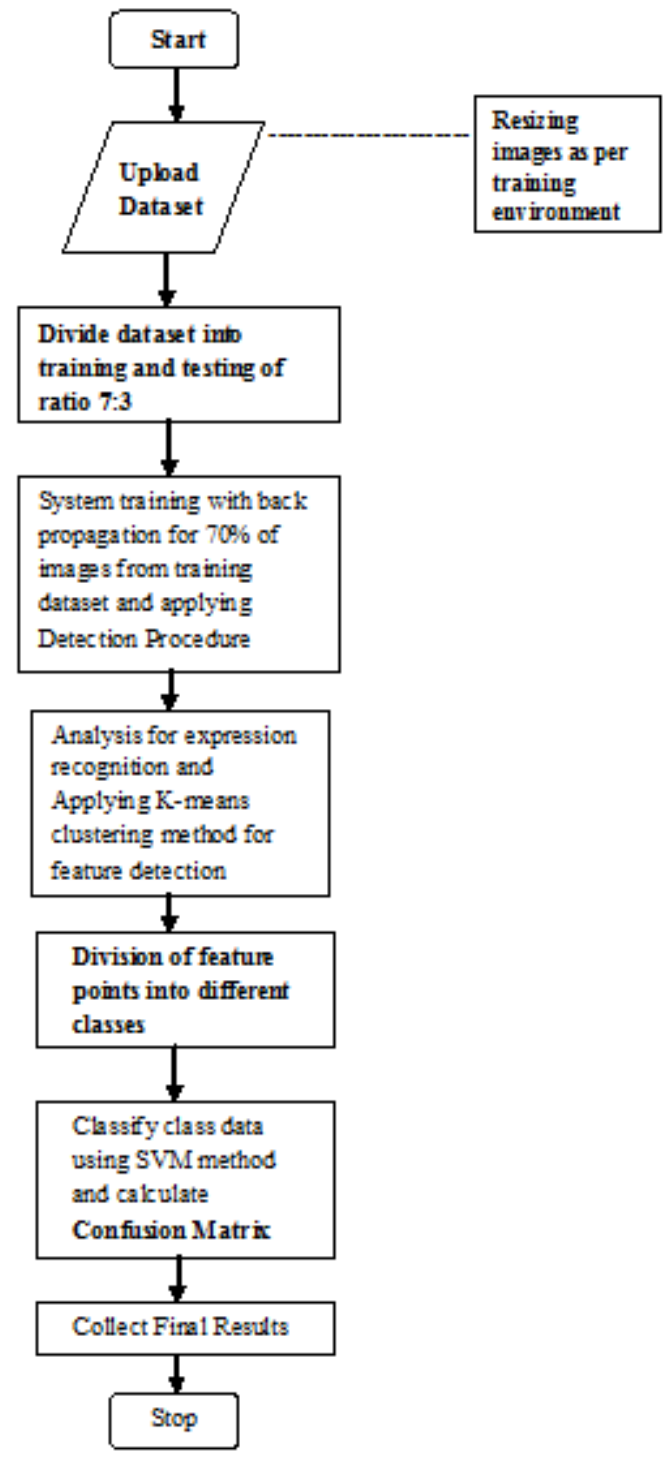

Figure 1: Proposed Algorithm

The above figure shows the proposed algorithm. We can see that upload dataset is the first step in which the recognised image dataset is uploaded which is then divided into training and testing sets of 7:3 ratio. The divided data set is then trained with back propogation algorithm and the detection procedure is applied through it thereby determining the six basic facial expression mentioned earlier in the abstract which are happy, sad, fear, angry, neutral and surprise. After the above mentioned steps the analysis for expression recognition is done and after that $\mathrm{K}$-means clustering algorithm is used to detect the desired features. "In statistics and data mining, k-means clustering is a method of cluster analysis which aims to partition $\mathrm{n}$ observations into $\mathrm{k}$ clusters in which each observation belongs to the cluster with the nearest mean.Given a set of observations (x1, x2, ..., $\mathrm{xn}$ ), where each observation is a d-dimensional real vector, k-means clustering aims to partition the $n$ observations into $\mathrm{k}$ sets $(\mathrm{k}=\mathrm{n}) \mathrm{S}=\{\mathrm{S} 1, \mathrm{~S} 2, \ldots, \mathrm{Sk}\}$ so as to minimize the within-cluster sum of squares,

$$
\underset{\mathbf{S}}{\operatorname{argmin}} \sum_{i=1}^{k} \sum_{\mathbf{x} \in S_{i}}\left\|\mathbf{x}-\boldsymbol{\mu}_{i}\right\|^{2}
$$

where $\mu \mathrm{i}$ is the mean of points in $\mathrm{Si}$. 


\section{International Journal of Science and Research (IJSR) \\ ISSN (Online): 2319-7064 \\ Index Copernicus Value (2013): 6.14 | Impact Factor (2014): 5.611}

k-Means cluster analysis achieves this by partitioning the data into the required number of clusters by grouping records so that the euclidean distance between the record's dimensions and the clusters centroid (point with the average dimensions of the points in the cluster) are as small as possible. Cluster analysis is a method for automatically grouping data into a smaller number of subsets or clusters so that the records grouped are most statistically similar to each other based on the attributes of the data compared.

These features are then divided into various classes after which the support vector machine comes into play and thereafter a confusion matrix is calculated on the basis of it. Arrival of the confusion matrix leads to the result.

\section{Implementation Notes}

The proposed system is implemented on MATLAB R2015b as it provides image processing toolbox which contains various methods related to image compression, enhancement, processing etc... The main motive of our implementation was to minimize the code as much as possible. Figure 2 below shows the basic functionalities of our implementation.

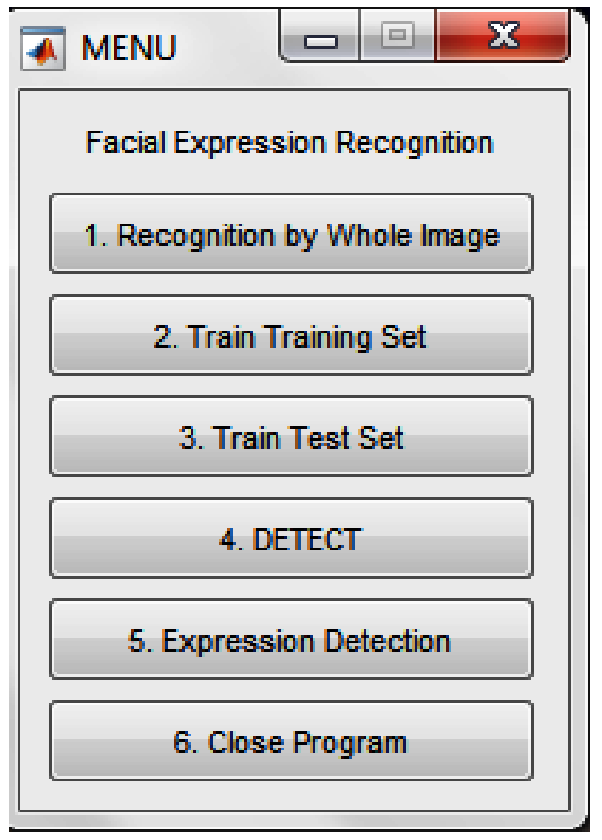

Figure 2: Menu

The first option which is stated as "Recognition by Whole Image" recognizes the image as whole. The second and third button/option trains the training as well as test set respectively. The six basic facial expressions mentioned in the abstract i.e. happy, fear, angry, sad, neutral and surprise are detected here as the part of facial expression recognition process. The detect option detects the image as the expression detection does the expression recognition. After the task has been completed the close program option can be selected to close the project.

The various phases of projects are shown below:

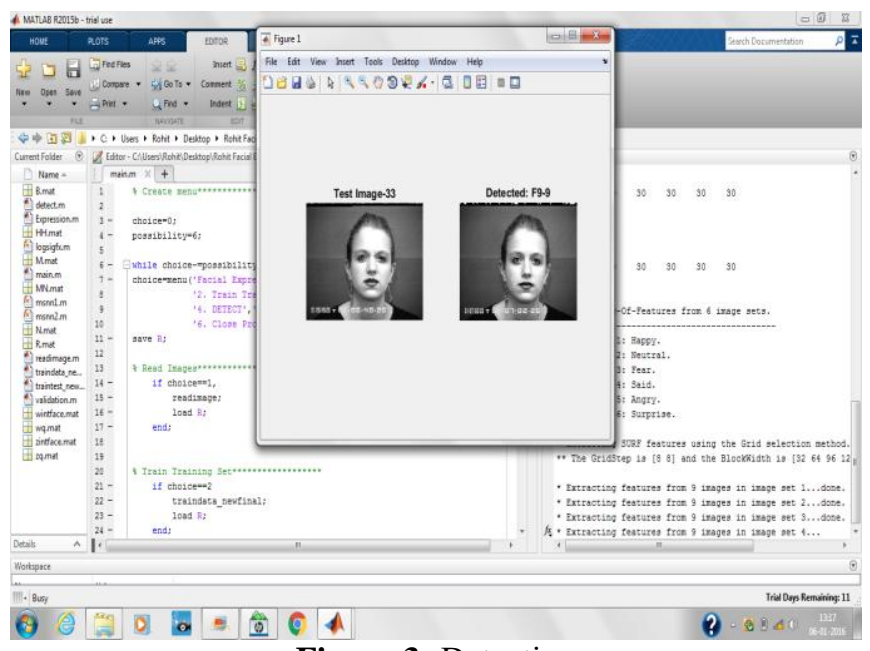

Figure 3: Detection

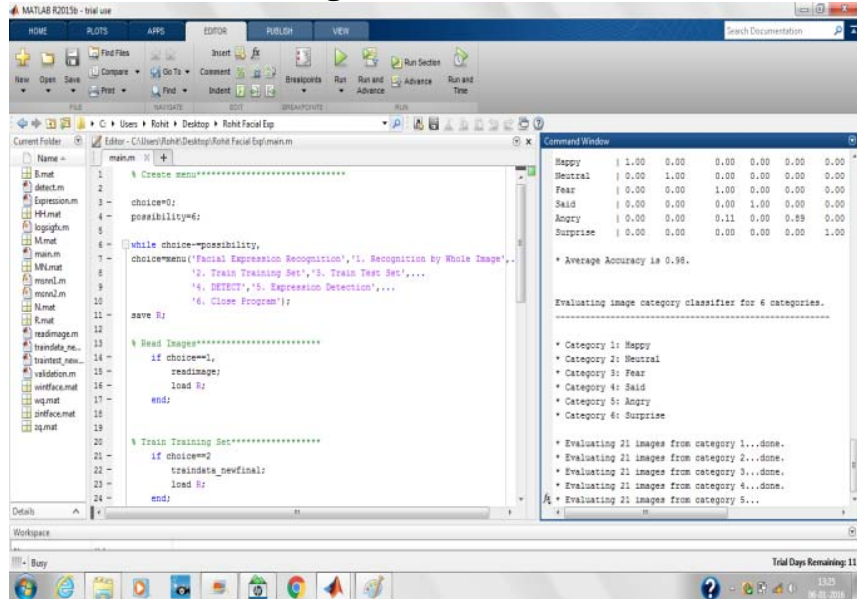

Figure 4: Overall Results

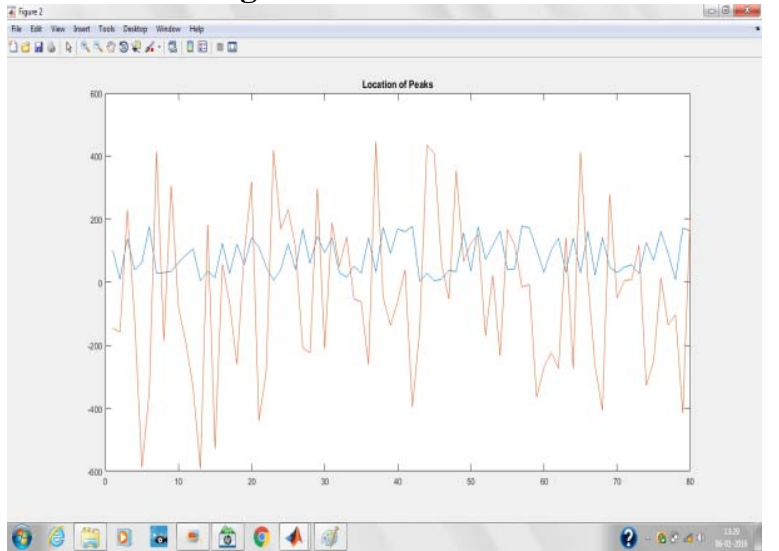

Figure 5: Graph Representing Features Intensity

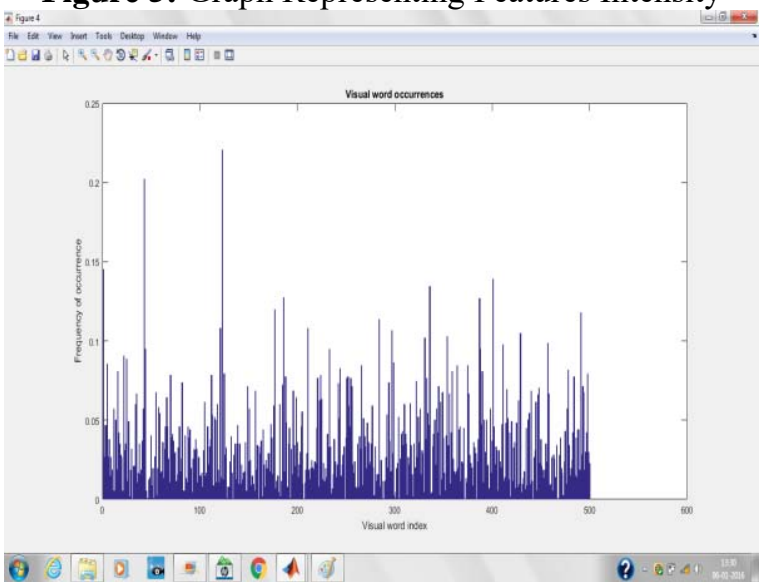

Figure 6: Visual word Occurrences 


\section{International Journal of Science and Research (IJSR) \\ ISSN (Online): 2319-7064}

Index Copernicus Value (2013): 6.14 | Impact Factor (2014): 5.611

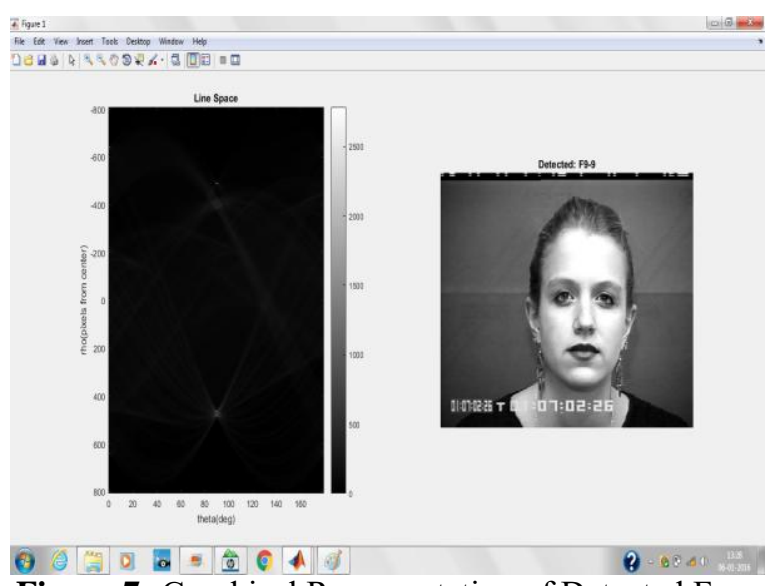

Figure 7: Graphical Representation of Detected Face Intensity with HSB

\section{Experimental Results}

In the previous section we analyzed the implementation process of our proposed work. In this section we will focus on the experimental results of our work. The proposed work was experimented on Cohn-Kanade dataset of images. The Cohn-Kanade AU-Coded Facial Expression Database is for reseaech in automatic facial image recognition, analysis and synthesis. It is available in two versions out of which version 1 includes 486 sequences from 97 posers. Each sequence of it starts with neutral expression and proceeds to a peak expression. The peak expression for each and every sequence is fully FACS (Facial Action Coding System) coded and given an emotion label. Version 2 is referred to as $\mathrm{CK}+$ includes both posed and non-posed expressions and additional types of metadata. For posed expressions the count of sequences increases from the initial release by $22 \%$ and the number of subjects by $27 \%$. As compared to Kai-Tai Song and Shuo-Cheng Chien's technique which is based on mixture of basic facial expressions and intensities our technique shows an increase of $3 \%$ which is quite satisfactory on its own thereby making our system accurate to $98 \%$.

\section{Conclusion}

Facial recognition has become a core area in today's world and is increasing its importance day by day as there are many applications in which it can be used. Our technique comprises of one of the most important parameter which is clustering due to which an accuracy of $98 \%$ is achieved thereby making it good with respect to recognition in various expressions. Future scope can include various other feature extraction techniques thereby making it more powerful with face at different angles and also with different light intensities and on other recognized image databases.

\section{References}

[1] Kai-Tai Song and Yi-Wen Chen "A Design for Integrated Face and Facial Expression Recognition" 978-1-61284-972-0/11 C2011 IEEE, pp.4306-4311.

[2] B. Lucas and T. Kanade, "An Iterative Image Registration Technique with an Application to Stereo
Vision," in Proc. of International Joint conference on Artificial Intelligence, 1981, Canada, pp. 674-679.

[3] Mingwei Huang and Zhen Wang, Zilu Ying Facial Expression Recognition Using Stochastic Neighbor Embedding and SVMs 978-1-61284-471-8/11 C2011 IEEE. Pp. 671-674.

[4] Nazil Perveen, Shubhrata Gupta, and Kesari Verma, "Facial Expression Recognition Using Facial Characteristic Points and Gini Index" 978-1-4673-04559/12 (C2012 IEEE.

[5] Huiquan Zhang, Sha Luo and Osamu Yoshie "Facial Expression Recognition by Analyzing Features of Conceptual Regions" 978-1-4799-0174-6/13@2013 IEEE pp.529-534.

[6] Varsha Sarawagi and K.V. Arya "Automatic Facial Expression Recognition for Image Sequences" 978-14799-0192-0/13 C2013 IEEE pp. 278-282.

[7] Anagha S. Dhavalikar and Dr. R. K. Kulkarni "2014 International Conference on Electronics and Communication System (ICECS -2014)" pp.1-7.

[8] Kamarul Hawari, Bin Ghazali, Jie Ma, Rui Xiao, "An Innovative Face detection based on Skin Color Segmentation", International Journal of Computer Applications (0975-8887), Volume 34-No.2, November 2011.

[9] W. Zhang and G. Cao, "Group Rekeying for Filtering False Data in Sensor Networks: A Predistribution and Local Collaboration-Based Approach," Proceedings from the Conference of the IEEE Communications Society, 2005, pp. 503-514.

[10] GJ. Edwards, T.F. Cootes, and CJ. Taylor, "Face recognition using active appearance models", Proceedings of the European Conference on Computer Vision, 1998.

[11] Pooja Kamavisdar, Sonam Saluja, Sonu Agrawal "A Survey on Image Classification Approaches and Techniques" International Journal of Advanced Research in Computer and Communication Engineering Vol. 2, Issue 1, January 2013.

[12] Jipsa Kurian, V.Karunakaran "A Survey on Image Classification Methods" International Journal of Advanced Research in Electronics and Communication Engineering (IJARECE) Volume 1, Issue 4, October 2012.

[13] Kamarul Hawari Ghazali, Mohd Fais Mansor, Mohd. Marzuki Mustafa and Aini Hussain, "Feature Extraction Technique using Discrete Wavelet Transform for Image Classification" The 5th Student Conference on Research and Development -SCOReD 2007 11-12 December 2007, Malaysia.

[14]E. V. PuginaA. L. Zhiznyakov, "Classification Of Features Of Image Sequences" The International Archives of the Photogrammetry, Remote Sensing and Spatial Information Sciences, Volume XL-5/W6, 2015 Photogrammetric techniques for video surveillance, biometrics and biomedicine, 25-27 May 2015, Moscow, Russia.

[15] Xuan Zhou, Jiajun Wang, "Feature Selection for Image Classification Based on a New Ranking Criterion", Journal of Computer and Communications, 2015, 3, 74 79 Published Online March 2015 in SciRes. 